\title{
Comprehensive Review of Formulations Mentioned for Vatavyadhi Chikitsa in Classical Texts
}

\author{
Vikas Kumar Sharma
}

Associate Professor, Dept of Samhita Siddhant, Shobhit University, Kunwar Shekhar Vijendra Medical College \& Research Center, Gangoh, Saharanpur, U.P

\begin{abstract}
Vatavyadhi Chikitsa (treatment) deals with diseases particularly caused by Vata dosha. (base of body) Vatavyadhi in present era is the most prevalent disease. Now a days Vatavyadhi are mostly concerned with neurological system, musculoskeletal system, reticulo-endothelial system and further pervades to all other systems in the body. Vata is the only dosha which governs all the other entities of the body like Pitta, Kapha,(other doshas means base of body) and Dhatu (fundamental support of body) etc. Diseases caused by vata in its vitiated (imbalance) condition are called Vatavyadhi. So, it is important to know the formulations which are mentioned in Classical texts especially in Brihattrayi (collection of three books) in order to keep all the body functions in equilibrium.
\end{abstract}

Keywords: Vatavyadhi, Brihattrayee, Vata, Pitta, Kapha, formulations

\section{INTRODUCTION}

Ayurveda is an age old science which dates back to thousands of years.
Vata is the only dosha which is responsible for vitiation as the action of other doshas, dhatu malas (excreta) all are dependent on vata and our body is dependent on dosha, dhatu and malal. It is also said that Pitta and Kapha are lame without the involvement of vata ${ }^{2}$. Vatavyadhi are the most severe types of diseases which afflict the individuals. Now days the diseases pertaining to Vatavyadhis are rising day by day and such patients approaching an Ayurvedic physician to get rid of the ailment. A better compilation of formulations for vatavyadhi mentioned in Brihattrayi will be helpful in further treatment.

\section{MATERIALS AND METHODS}

This article is based on a comprehensive review of formulations mentioned for Vatavyadhi Chikitsa. Materials \& references related to this were taken from Brihattrayi i.e. Charak Samhita, Sushruta Samhita and Ashtang Hridaya.

\begin{tabular}{|l|l|l|l|l|}
\multicolumn{9}{|c}{ Table No.1 : Showing formulations mentioned in Charaka samhita vatavyadhi cikitsa } \\
\hline S.No: & Adhikarana & Formulation & $\begin{array}{l}\text { Single/ } \\
\text { compound } \\
\text { ingredients }\end{array}$ & Single/multiple indications \\
\hline 1 & C. Ci. $28 / 79$ & Masha tailam & C.I. & S. I. samkucite \\
\hline 2 & C. Ci. $28 / 113$ & Mustadiupanaha & C.I. & S. I. sarujam gatram \\
\hline 3 & C. Ci. $28 / 134$ & Nirgundi tailam & S. I. & M. I. nadi vrana, kushtha, vatamaya, pama, apaci \\
\hline 4 & C. Ci. $28 / 131-133$ & Rasnadi tailam & C.I. & $\begin{array}{l}\text { M. I. sira, majja, asthi gata vata, sarvanga, ekanga vata, } \\
\text { vepana, akshepaka, sula }\end{array}$ \\
\hline 5 & C. Ci. $28 / 136-138$ & Mulakadyam tailam & C.I. & S. I. vatanut \\
\hline 6 & C. Ci. $28 / 138,139$ & Panjcamula tailam & C.I. & M. I. sarvavata vikaranut, vata samsrshta kapha \\
\hline 7 & C. Ci. $28 / 140,141$ & Yavadi tailam & C.I. & S. I. mahavatairarta \\
\hline 8 & C. Ci. $28 / 144-146$ & Sahacaraditaila & C.I. & S. I. darune vatavyadhishu \\
\hline 9 & C. Ci. $28 / 146-148$ & Svadamshtra tailam & C.I. & S. I. sarvanilartishu \\
\hline 10 & C. Ci. $28 / 148-157$ & Bala tailam & C.I. & $\begin{array}{l}\text { M. I. svasa, kasa, jvara, hikka, chardi, gulma, kshata, } \\
\text { kshaya, pliha, sosha, apasmara, alakshmi, vatavyadhi }\end{array}$ \\
\hline 11 & C. Ci. $28 / 157-164$ & Amrtadyam tailam & C.I. & $\begin{array}{l}\text { M. I. ksheena virya, agni bala, sammudha cetasa, unmada, } \\
\text { arati, apasmara, vatavyadhi }\end{array}$ \\
\hline 12 & C. Ci. 28/165-166 & Rasna tailam & C.I. & S. I. anilartinut \\
\hline
\end{tabular}


Vikas Kumar Sharma. Comprehensive review of formulations mentioned for vatavyadhi chikitsa in classical texts. International Journal of Research and Review.

\begin{tabular}{|c|c|c|c|c|}
\hline \multicolumn{5}{|c|}{ Table 1 Continued... } \\
\hline 13 & C. Ci. $28 / 167-169$ & Mulakadyam tailam & C.I. & S. I. ugran vatatmakan gadan \\
\hline 14 & C. Ci. $28 / 170-171$ & $\begin{array}{l}\text { Vrshamuladi } \\
\text { tailam }\end{array}$ & C.I. & $\begin{array}{l}\text { M. I. vayuna bhagne dadyat } \\
\text { jarjarite }\end{array}$ \\
\hline 15 & C. Ci. $28 / 172-175$ & Mulaka tailam & C.I. & $\begin{array}{l}\text { M. I. pleeha, mutragraha, svasa, kasa, maruta roga, } \\
\text { varnayurbalavardhanam }\end{array}$ \\
\hline 16 & C. Ci. $28 / 176$ & $\begin{array}{l}\text { Yavakola } \quad \text { kulathadi } \\
\text { tailam }\end{array}$ & C.I. & S. I. sarvavatamayapaham \\
\hline 17 & C. Ci. $28 / 177$ & Lasuna tailam & S. I. & M. I. vatanut, vandhyatvahara \\
\hline 18 & $\begin{array}{l}\text { C. Ci. } 28 / 179 \\
\text { (jvaradhikarana) }\end{array}$ & $\begin{array}{l}\text { Agurvadyam } \\
\text { tailam }\end{array}$ & C.I. & M. I. \\
\hline 19 & $\begin{array}{l}\text { C. Ci. } 28 / 180 \\
\text { (rasayanadhikarana) }\end{array}$ & Cyavanaprasa & C.I. & M. I. \\
\hline 20 & $\begin{array}{l}\text { C. Ci. } 28 / 84 \\
\text { (Susruta vatavyadhi } \\
\text { cikitsa) }\end{array}$ & $\begin{array}{l}\text { Tilvaka ghrta and satala } \\
\text { siddha ghrta }\end{array}$ & C.I. & S. I. \\
\hline 21 & C.Ci. $28 / 96$ & $\begin{array}{l}\text { Sita-kasmarya- } \\
\text { madhuka yukta- paya. }\end{array}$ & C.I. & $\begin{array}{l}\text { M. I. vata-vyadhi like garbha- sosha due to vata, bala-sosha } \\
\text { due to vata etc }\end{array}$ \\
\hline
\end{tabular}

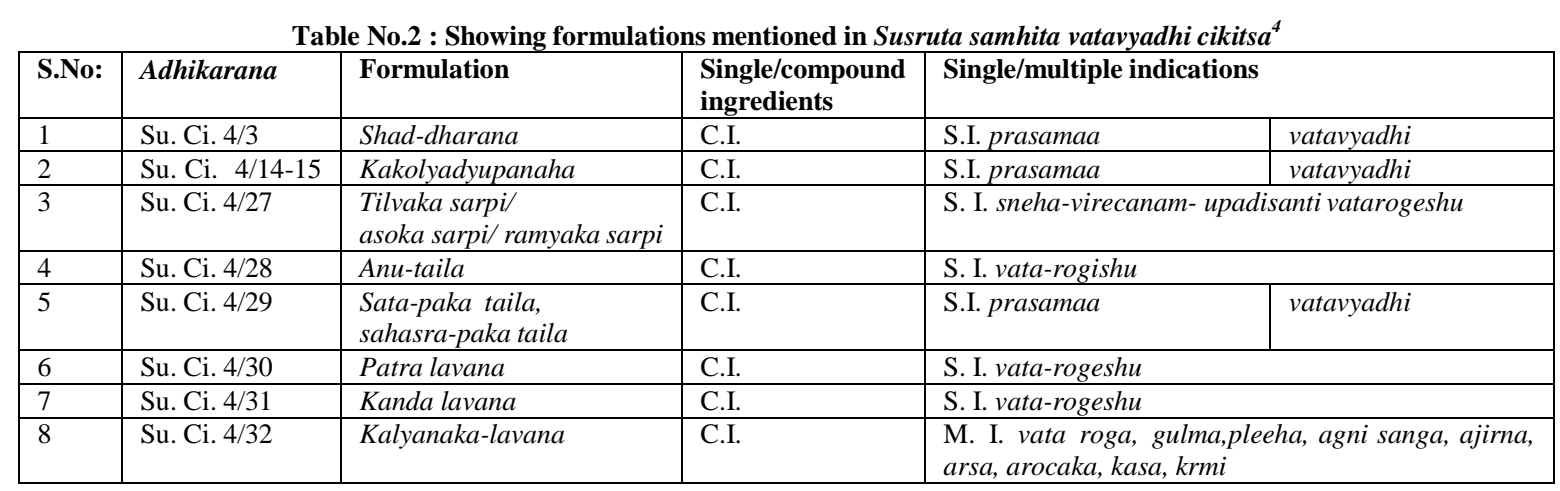

\begin{tabular}{|c|c|c|c|c|}
\hline \multicolumn{5}{|c|}{ Table no 3: showing formulations mentioned in Ashtanga Hrdaya vatavyadhi cikitsa ${ }^{5}$} \\
\hline S.No: & Adhikarana & Formulation & $\begin{array}{l}\text { Single/ } \\
\text { compound } \\
\text { ingredients }\end{array}$ & Single/multiple indications \\
\hline 1 & $\begin{array}{l}\text { A. H. Ci. } 21 . \\
\text { 21. (C.Ci.28/96) }\end{array}$ & $\begin{array}{l}\text { Sita-kasmarya- } \\
\text { madhuka yukta- } \\
\text { paya. }\end{array}$ & C.I. & $\begin{array}{l}\text { M. I. vata-vyadhi like garbha- } \\
\text { sosha due to vata, bala-sosha dueto vata etc }\end{array}$ \\
\hline 2 & $\begin{array}{l}\text { A. H. Ci. } 21.22 \text { (C. Ci. } \\
\text { 28/79) }\end{array}$ & Masha tailam & C.I. & S. I. samkucite \\
\hline 3 & A. H. Ci. $21.26-27$ & $\begin{array}{l}\text { Vidaryadi gana } \\
\text { ghrta }\end{array}$ & C.I. & S. I. vayurvapnoti sahasaiva \\
\hline 4 & A. H. Ci. 21.31 .5 & $\begin{array}{l}\text { Sauvarcaladi } \\
\text { sarpi }\end{array}$ & C.I. & S. I. cale adhike \\
\hline 5 & A. H. Ci. $21.32-33$ & $\begin{array}{l}\text { Tilvaka sarpi/ } \\
\text { ramyaka/asoka }\end{array}$ & C.I. & $\begin{array}{l}\text { M. I. dushtan vatan, ekanga-vata, } \\
\text { sarvanga-vata, yonivyapat, gulma,vrdhma, udaram }\end{array}$ \\
\hline 6 & $\begin{array}{l}\text { A. H. Ci. } 21 . \\
35-37\end{array}$ & $\begin{array}{l}\text { Tumburunydi } \\
\text { curna }\end{array}$ & C.I. & $\begin{array}{l}\text { M. I. hrd-parsva arti, apatantraka, } \\
\text { sleshma-pavana hrdroga }\end{array}$ \\
\hline 7 & $\begin{array}{l}\text { A. H. } \\
\begin{array}{l}\text { Ci. } 21.48 \\
\text { Susruta } \\
\text { cikitsa) }\end{array}\end{array}$ & Shad-dharana & C.I. & S. I. vatavyadhi prasamana \\
\hline 8 & A. H. Ci. 21.49 & Vyoshadi guggulu & C.I. & M. I. meda-sleshma-amavatajan \\
\hline 9 & A. H. Ci. 21.55 & $\begin{array}{l}\text { Sahacaradi } \\
\text { kashaya }\end{array}$ & C.I. & $\begin{array}{l}\text { S. I. vata-pidita deha gati - druta } \\
\text { vilambitago bhavaticchaya }\end{array}$ \\
\hline 10 & A. H. Ci. 21.56 & Rasna ghrtam & C.I. & S. I. vatarogaharam param \\
\hline 11 & A. H. Ci. $21.57-60$ & $\begin{array}{l}\text { Guggulu-tiktaka } \\
\text { ghrta }\end{array}$ & C.I. & $\begin{array}{l}\text { M. I. prabalam sameeram, sandhyasthi majjagata vata, } \\
\text { kushtha, nadivrana, arbuda, bhagandara, gandamala, } \\
\text { jatrurdhva sarvagada, gulma, gudotha, meha, yakshma, aruci, }\end{array}$ \\
\hline 12 & $\begin{array}{l}\text { A. H. Ci. } 21 . \\
64-65\end{array}$ & $\begin{array}{l}\text { Prasaranyadi } \\
\text { taila }\end{array}$ & C.I. & S. I. sarva marutamayanasanam \\
\hline 13 & A. H. Ci. 21.64 & $\begin{array}{l}\text { Pinyaka- } \\
\text { panjcamula tailam }\end{array}$ & C.I. & S. I. vata nasyeyu: sleshmayukta \\
\hline 14 & A. H. Ci. 21. 67-68 & Sahacaradi taila & C.I. & $\begin{array}{l}\text { M. I. vatan, kampa, akshepa, stambha, sosha, gulma, unmada, } \\
\text { pinasam, yoniroga }\end{array}$ \\
\hline 15 & A. H. Ci. 21. 69-72 & $\begin{array}{l}\text { Sahacaradi taila } \\
\text { (2) }\end{array}$ & C.I. & $\begin{array}{l}\text { M. I. krcchran-anilamayan, } \\
\text { vatakundalika, unmada, gulma, bardhma, }\end{array}$ \\
\hline 16 & $\begin{array}{l}\text { A. H. Ci. 21. - 73-81 } \\
\text { (C. Ci. } 28 / 148-157 \text { ) }\end{array}$ & Bala tailam & C.I. & $\begin{array}{l}\text { M. I. svasa, kasa, jvara, hikka, chardi, gulma, kshata, kshaya, } \\
\text { pliha, sosha, apasmara, alakshmi, vatavyadhi }+ \text { dushtan } \\
\text { vatanasu santi, nayeyur-vandhya nari } \\
\text { putrabhajasca kuryu }\end{array}$ \\
\hline
\end{tabular}


Vikas Kumar Sharma. Comprehensive review of formulations mentioned for vatavyadhi chikitsa in classical texts. International Journal of Research and Review.

\section{DISCUSSION}

In Charaka Samhita vata-vyadhi adhyaya many of the formulations are not named, and for convenience of the review, those formulations have given names on the basis of primary ingredient. Among 21 formulations 17 are oil preparations. This observation points towards the consideration of oil as the best alleviating medicine in vata vitiation. Formulations with more than one ingredient as well as single drug administration are explained. Medicines with one/multiple indications are also seen. For example Masha tailam a compound drug formulation has only one indication i.e.; samkucite. At the same time compound drug formulation like Bala tailam has multiple indications such as svasa, kasa etc. On the other hand single drugs explained with multiple indications eg. Nirgundi tailam for nadi vrana, kushtha, vatamaya etc. but no single drug is mentioned just for vata-vyadhi alone in Caraka samhita. Caraka has adopted Agurvadyam tailam from jvaradhikara, Cyavanaprasa from rasayana-adhikara and Tilvaka sarpi and Satala sarpi are also seen in Susruta samhita vata-vyadhi cikitsa.

In Sushruta samhita vata-vyadhi cikitsa has mentioned about 8 formulations and all are named. Among eight formulations 3 are lavana preparations. This is a unique contribution of Susruta samhita for vata-vyadhi cikitsa. No single drug formulations are explained. But among eight formulations seven have only one indication i.e.; vata-vyadhi.

In Ashtanga hrdaya also does not mention any single drug formulation in vata-vyadhi cikitsa. But eight out of sixteen formulations are having only vata-vyadhi indication. Ashtanga hrdaya has taken Sitakasmarya-madhuka yukta paya, Masha tailam and Bala tailam from Caraka samhita vata-vyadhi cikitsa and mentioned Shaddharana curna of Sushruta-samhita. For bala-tailam two more indications have been mentioned by Ashtanga hrdaya.

\section{CONCLUSION}

We came across many patients of vatavyadhi in routine OPD. So above compilation of formulations for vatavyadhi in one place will be very helpful for the treatment of these diseases for the Doctors and Research Scholars.

\section{Acknowledgement: None}

\section{Conflict of Interest: None}

\section{Source of Funding: None}

\section{REFERENCES}

1. Sushruta Samhita, by Dr Sh Bhaskar Goivnd ji Ghanekar,published by Motilal Bnarsidas Delhi, Fifith Edition, chapter no 15/3,page no55

2. Padarth vigyan,by Dr K.V.L Narasimhacharyulu, published by Chowkhamaba Krishandas Academy, Sixth edition ,page no 50.

3. Acharya JT, editor. Caraka samhita of Agnivesa, with Ayurveda-deepika commentary of Cakrapani Datta. Reprint ed. Varanasi (India): Chaukhambha Krishnadas Academy; 2010. p. 675.

4. Acharya JT, editor. Susruta samhita of Susruta, with Nibandha samgraha commentary of Dalhana. Reprint ed. Varanasi (India): Chaukhambha Surbharati Prakashan; 2010. p. 448.

5. Paradakara HSS, editor. Ashtanga hrdaya of Vagbhata, with Sarvanga sundara commentary of Arunadatta and Ayurveda rasayana commentary of Hemadri. Reprint ed. Varanasi (India): Chaukhambha Sanskrit sansthan, 2009. p. 517.

How to cite this article: Sharma VK. Comprehensive review of formulations mentioned for vatavyadhi chikitsa in classical texts. International Journal of Research and Review. 2021; 8(8): 182-184. DOI: https://doi. org/10.52403/ijrr.20210825 